\title{
Caracterización clínico-genético- molecular de 45 pacientes chilenos con Síndrome de Prader Willi
}

\author{
Fanny Cortés $\mathbf{M}^{\mathbf{1}}, \mathbf{M}$. Angélica Alliende $\mathbf{R}^{\mathbf{1}, \mathbf{a}}$, Andrés Barrios $\mathbf{R}^{\mathbf{1}, \mathbf{2}}$ \\ Bianca Curotto L ${ }^{1, b}$, Lorena Santa María V1,c, Ximena Barraza \\ $\mathrm{O}^{3}$, Ledia Troncoso $\mathrm{A}^{2}$, Cecilia Mellado $\mathrm{S}^{4,6}$, Rosa Pardo $\mathrm{V}^{1,6,7}$. \\ Clinical, genetic and molecular features \\ in 45 patients with Prader-Willi \\ Syndrome
}

Background: Prader-Willi syndrome (PWS) is a neurogenetic disease characterized by neonatal hypotonia, retarded mental and motor development, hypogonadism, hyperphagia, morbid obesity and dysmorphic facial features. It has an incidence of 1:12.000-15.000 newborns and is caused by abnormalities in genes located in 15q11q13. PWS is one of the most frequent genetic disorders and microdeletion syndromes. It is also the most common cause of obesity from genetic origin and it was the first disease in which imprinting and uniparental disomy were recognized as cause of genetic disorders. Seventy to seventy five percent of PWS cases are due to 15q11q13 deletions, 20-25\% to uniparental disomy and 1\% to mutations in the imprinting center. Aim: To analyze the clinical, genetic and molecular features of patients with PWS, seen at one institution. Patients and methods: Retrospective review of 45 patients (27 males) with PWS seen at the Genetics Outpatient Clinic at INTA. Results: Twenty three (51.1\%) patients had a delection, 13 (28.9\%) patients did not have a deletion. In nine patients, fluorescence in situ hybridization (FISH) study was not performed, therefore the presence of deletion was unknown. The clinical score was 8 points for patients younger than 3 years $(n=11)$ and 11.5 points for patients older than 3 years $(n=34)$; for patients aged 12 months or less, the clinical score was 7 points. Mean clinical score was 11 points for patients with deletion and 10 points for patients without deletion. Conclusions: Most patients with PWS have a deletion; the phenotype depends on age and the clinical score is useful for Chilean patients with PWS (Rev Méd Chile 2005; 133: 33-41).

(Key-words: Abnormalities, multiple; Mental retardation; Prader-Willi syndrome)

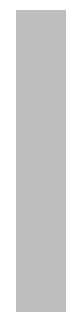

\footnotetext{
Recibido el 17 de junio, 2004. Aceptado en versión corregida el 19 de octubre, 2004. Nacidos, Hospital Clínico, Universidad de Chile

aMagíster en Ciencias Biológicas

bTecnólogo Médico

'Bioquímica, alumna de Doctorado en Ciencias, Universidad de Chile
}

${ }^{1}$ Unidad de Genética y Enfermedades Metabólicas, INTA, Universidad de Chile. ${ }^{2}$ Servicio de Neurología Infantil Hospital San Borja Arriarán. ${ }^{3}$ Hospital Regional de Valdivia, Valdivia. ${ }^{4}$ Sección Genética, Hospital Clínico de la Universidad Católica de Chile. ${ }^{5}$ Sección de Genética, Hospital Clínico, Universidad de Chile. ${ }^{6}$ Sección de Genética, Hospital Dr. Sótero del Río. ${ }^{7}$ Unidad de Recién

Correspondencia a: Dra. Fanny Cortés M. Unidad de Genética y Enfermedades Metabólicas. Instituto de Nutrición y Tecnología de los Alimentos (INTA), Universidad de Chile. Macul 5540, Santiago, Chile. E-mail: fcortes@inta.cl 
$\mathrm{E}^{1}$ Síndrome de Prader Willi (SPW) es una enfermedad neurogenética compleja y multisistémica, caracterizada por hipotonía neonatal, retraso del desarmollo psicomotor, hipogonadismo hipogonadotrófico, hiperfagia, obesidad mórbida y dismorfias craneofaciales características (disminución del diámetro biparietal, ojos almendrados, boca triangular), entre otros elementos fenotípicos (Figura 1). Su incidencia se estima en 1:12.000-15.000 recién nacidos (RN) y es producida por diversas alteraciones de genes ubicados en el cromosoma 15 en la región 15q11q131. La gran mayoría de los individuos con SPW son los únicos afectados en sus familias y por lo tanto se considera una afección esporádica, sin un riesgo aumentado de recurrencia, salvo rarísimas excepciones como se verá más adelante ${ }^{2}$.

El SPW se considera una de las afecciones genéticas y uno de los síndromes de microdeleción más frecuentes; es la causa más común de obesidad de origen genético y fue la primera afección en la que se reconoció la microdeleción, la impronta genómica y la disomía uniparental en su origen ${ }^{3-6}$. El rol de la impronta y de la disomía uniparental se estableció al demostrar que una afección genética con un fenotipo completamente diferente como es el Síndrome de Angelman, se producía cuando una microdeleción también comprometía al segmento 15q11q13, pero en el cromosoma de origen materno o bien cuando existía una disomía uniparental paterna. Lo anterior significa que algunos genes ubicados a nivel de 15q11q13 tienen expresión diferencial dependiendo de si son alelos heredados de la madre o del padre. El SPW se produce por falta de expresión de genes del alelo paterno ubicados en 15q11q13; estos genes son normalmente activos en el alelo heredado desde el padre e inactivos en los heredados desde la madre. Esta diferencia entre ambos alelos se produce por un evento epigenético de metilación diferencial, estando, normalmente, el alelo materno altamente metilado. Este patrón diferencial de metilación es finalmente el que determina en este caso la impronta genómica que por definición se refiere al mecanismo por el cual algunos genes se modifican de diferentes formas, dependiendo del género del progenitor del que son heredados, determinando la expresión diferente de ambos alelos ${ }^{3-6}$.

Hasta ahora, no se conocen exactamente los genes responsables de la expresión del fenotipo característico del síndrome ${ }^{4}$. Se han descrito va-

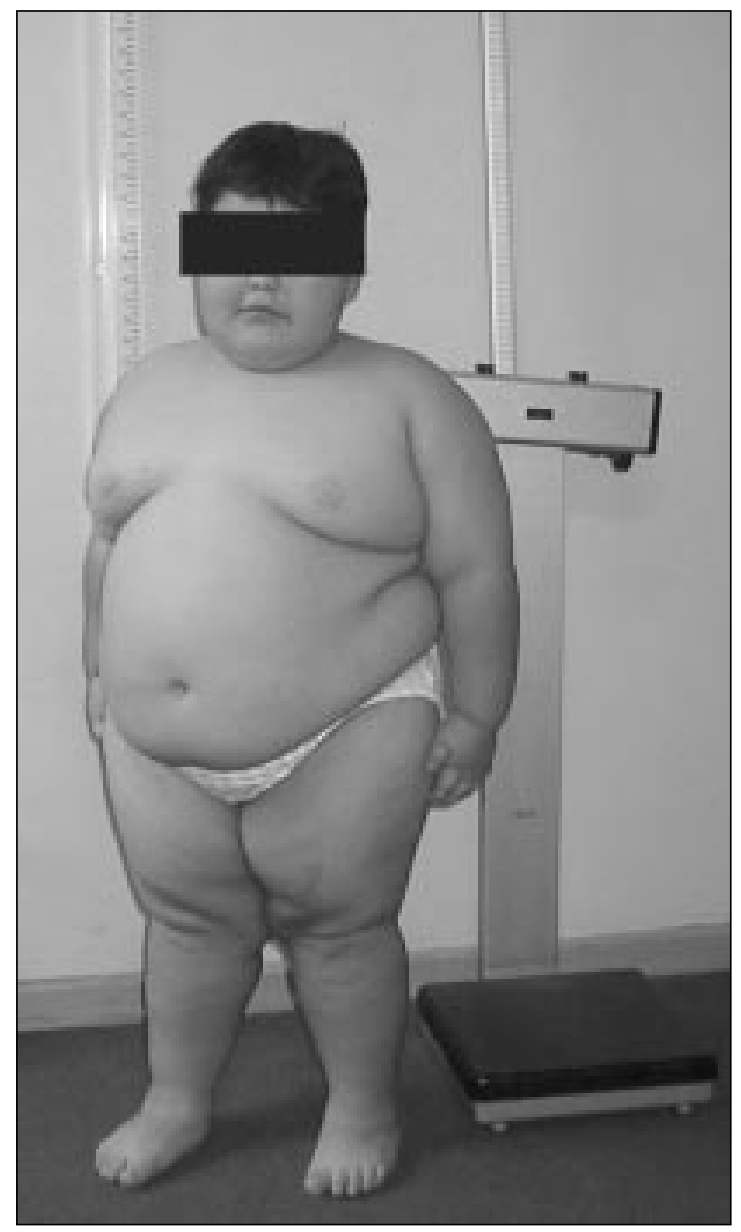

Figura 1. Paciente de 8 años con Síndrome de Prader Willi.

rios genes «mprintados»y «no imprintados»en la región cromosómica involucrada en la afección y, para la mayoría de ellos, aún no está claro como la no expresión de un gen se relaciona con la patogénesis. Entre los genes más importantes están el gen SNRPN y el gen P; el gen SNRPN que ha sido el gen más estudiado, y que se estima sería el responsable de la mayoría de las características fenotípicas del SPW, codifica para una ribonucleoproteína pequeña, que cumple un rol en el procesamiento del ARN, específicamente en el splicing génico, para que posteriormente ocurra la síntesis de proteínas. Se ha demostrado que SNRPN está «mprintado»en el cerebro, que es el foco de la mayoría de las manifestaciones de la afección ${ }^{7}$. El gen SNRPN es el que con mayor frecuencia se utiliza con fines diagnósticos. Por 
otra parte, se sabe que el gen $P$ es un gen no «mprintado»que codifica para albinismo tirosinasa positivo y cuya deleción se ha asociado con la hipopigmentación que se ve en alrededor de $30 \%$ de los individuos con SPW 8 .

Hasta ahora, se han identificado tres mecanismos de producción en el SPW: 70-75\% de los casos se producen por una pequeña deleción en el cromosoma 15 de origen paterno entre las bandas $15 q 11$ y 15q13, en la gran mayoría de los casos se produce una deleción de $4-\mathrm{Mb}$, aunque se ha descrito un pequeño número de pacientes con una deleción de menor tamaño ${ }^{7}$; de los restantes individuos con SPW, 20-25\% se producen por disomía uniparental (DUP) materna, es decir, son individuos que han heredado dos copias del segmento 15q11q13 de la madre y ninguna del padre. De estos dos grupos de pacientes, alrededor de 2-5\% tienen una deleción o una DUP producto de una alteración cromosómica estructural que involucra al cromosoma 15 en el segmento crítico para esta afección. Finalmente, en el origen del SPW se ha podido determinar que $1 \%$ de los pacientes no tiene deleción ni DUP sino que el SPW es resultado de mutaciones del centro regulador de la impronta. Todas las familias descritas en las que ha habido recurrencia de la afección han demostrado tener mutaciones del centro regulador de la impronta ${ }^{8}$.

Existen criterios de consenso (Holm) para el diagnóstico clínico de esta afección ${ }^{9}$ (Tabla 1). Sin embargo, siempre se debe realizar la confirmación diagnóstica. Esta confirmación diagnóstica se puede hacer por estudio de citogenética con bandeo

Tabla 1. Criterios diagnósticos de H olm

Criterios mayores

1. Hipotonía neonatal e infantil de tipo central. Con dificultad de la succión, que mejora con la edad.

2. Problemas de alimentación, con antecedente de requerir gavage, SNG u otra técnica especial de alimentación

3. Inicio de ganancia ponderal muy rápida entre los 12 meses y los 6 años, lo que determina obesidad de tipo central

4. Dismorfias faciales características: disminución del diámetro biparietal, ojos almendrados, fisuras labiales hacia abajo.

5. Hipogonadismo (hipoplasia genital: labios menores y clítoris en las mujeres, escroto hipoplásico y criptorquidia en los hombres / pubertad incompleta o retardada / infertilidad

6. Retraso del desarrollo / retraso mental leve a moderado / trastornos múltiples del aprendizaje

7. Hiperfagia

8. Defecto citogenético o molecular: deleción 15q11q13, disomía uniparental materna 15 o test de metilación alterado.

Criterios menores

1. Disminución de los movimientos fetales y letargia que mejora con la edad

2. Problemas conductuales característicos: rabietas, conductas obsesivo compulsivas, testarudez, rigidez, cleptomanía y mitomanía.

3. Trastorno del sueño/apnea del sueño

4. Talla baja

5. Hipopigmentación

6. Manos y pies pequeños para la talla

7. Manos angostas con borde cubital recto

8. Estrabismo, miopía

9. Saliva gruesa, viscosa

10. Defectos de la articulación del lenguaje

11. Grataje de la piel (picking)

Cada criterio mayor tiene valor de 1 punto. Cada criterio menor tiene valor de 0,5 puntos

Para el diagnóstico se requiere:

- En niños menores de 3 años: 5 puntos (mínimo 4 criterios mayores)

- En niños mayores de 3 años: 8 puntos (mínimo 5 criterios mayores) 
de alta resolución (confirma el diagnóstico sólo en $5 \%$ de los casos), estudio de hibridación in situ con fluorescencia (FISH) (confirma el diagnóstico en $70-75 \%$ de los casos) y la prueba de metilación (positiva en 99\% de los casos).

$\mathrm{Si}$ bien los criterios diagnósticos publicados por Holm ${ }^{9}$ han probado su utilidad, en la actualidad hay evidencia en la literatura, que un porcentaje importante, cercano al $16 \%$, de pacientes con diagnóstico molecular de SPW, analizados retrospectivamente, no calzan con los criterios de diagnóstico clínico. Por lo tanto, se ha sugerido usar criterios menos estrictos de manera de incluir a un mayor número de individuos susceptibles de estar afectados ${ }^{10}$.

El estudio cromosómico con bandeo de alta resolución permite detectar otras posibles alteraciones cromosómicas concomitantes y sólo un muy bajo porcentaje de los pacientes con deleción ${ }^{11}$; el estudio con FISH permite pesquisar a todos los pacientes con deleción y el estudio de metilación permite detectar a los pacientes con deleción, DUP o mutaciones del centro regulador de la impronta.

En el laboratorio de citogenética molecular del Instituto de Nutrición y Tecnología de los Alimentos (INTA), se han implementado todas estas técnicas diagnósticas y en el CEDINTA (Centro Diagnóstico del INTA) existe un equipo multidisciplinario de seguimiento de estos pacientes el que, además, asesora a la agrupación chilena de padres de niños con SPW.

Objetivo. Caracterizar desde el punto de vista clínico, genético y molecular a los 45 pacientes con SPW que se controlan en el policlínico de genética del INTA, validar en nuestra población los criterios de diagnóstico clínico, describir si existe una relación fenotipo-genotipo y establecer la mejor metodología de estudio diagnóstico para pacientes chilenos con SPW.

\section{SujETOS Y MÉTODOS}

Se evaluaron todas las características clínicas de cada uno de los 45 pacientes con SPW que se encuentran en control a la fecha de esta publicación, en el policlínico de Genética del CEDINTA y se determinó la forma en que se estableció el diagnóstico de certeza. Como grupo control se consideró a 23 individuos en los que clínicamente se sospechó un SPW y cuyo estudio de metilación resultó normal, con lo cual se descartó el diagnóstico planteado.

La evaluación de los criterios diagnósticos de Holm, en pacientes y controles, se realizó por un solo examinador, una vez obtenida la confirmación diagnóstica o en el momento de la primera consulta en el INTA, cuando se trata de pacientes derivados con diagnóstico confirmado.

Los exámenes de citogenética convencional, FISH y prueba de metilación fueron realizados en el Laboratorio de Citogenética Molecular del INTA, de acuerdo a técnicas estandarizadas y previamente publicadas $^{12}$, en aquellos pacientes que no los tenían al momento de la primera consulta. Dos pacientes fueron derivados a CEDINTA con estudios de FISH realizados en otros centros y cuatro con estudios de metilación realizados en el INTA, pero solicitados desde otros centros, todos ellos fueron derivados desde la agrupación chilena para el Síndrome de Prader Willi.

\section{RESULTADOs}

Un total de 45 pacientes con SPW se encuentran, al momento de esta publicación, en control en el policlínico de Genética de CEDINTA. Un resumen de las características generales de los pacientes se presenta en la Tabla 2 y un resumen de las características fenotípicas de acuerdo a los criterios de Holm se presenta en la Tabla 3.

La distribución por sexo fue de 27 (60\%) mujeres y 18 (40\%) hombres. La edad de diagnóstico en promedio fue de 36 meses, con un rango entre 10 días y 20 años, con una clara tendencia a disminuir la edad de diagnóstico en los últimos años. La mediana de la edad fue de 12 meses; con una moda, edad a la que con mayor frecuencia se hizo el diagnóstico, de 1 mes.

El grupo control estuvo constituido por 23 individuos, 11 hombres y 12 mujeres con edad promedio de 68 meses con un rango entre 4 meses y 15 años, todos con prueba de metilación normal.

En relación con el estudio diagnóstico realizado, este fue variable: 6 pacientes $(13,3 \%)$ tenían sólo cariograma, 4 (9\%) sólo prueba de metilación, $6(13,3 \%)$ cariograma y FISH, $5(11,1 \%)$ 
Tabla 2. Resumen de características generales de los 45 pacientes con SPW incluidos en el estudio

\begin{tabular}{|c|c|c|c|c|c|c|c|c|c|}
\hline \multirow[t]{2}{*}{ Paciente } & \multirow[t]{2}{*}{ Sexo } & \multirow{2}{*}{$\begin{array}{c}\text { Edad } \\
\text { Dg }\end{array}$} & \multirow{2}{*}{$\begin{array}{l}\text { Edad } \\
\text { Score }\end{array}$} & \multirow[t]{2}{*}{ Metilació } & \multirow[t]{2}{*}{ FISH } & \multirow[t]{2}{*}{ Cariograma } & \multicolumn{3}{|c|}{ Score de Holm (puntos) } \\
\hline & & & & & & & $\begin{array}{l}\text { Criterios } \\
\text { mayores }\end{array}$ & $\begin{array}{l}\text { Criterios } \\
\text { menores }\end{array}$ & Total \\
\hline 1 & $\mathrm{M}$ & $17 a$ & $20 \mathrm{a}$ & no & no & 46,XY,del (15) (q11q13) & 7 & 3,5 & 10,5 \\
\hline 2 & $\mathrm{~F}$ & $1 \mathrm{a}$ & $14 \mathrm{a}$ & no & no & 46,XX,del (15) (q11q13) & 8 & 5 & 13 \\
\hline 3 & $\mathrm{~F}$ & $1 \mathrm{a} 6 \mathrm{~m}$ & $6 \mathrm{a} 2 \mathrm{~m}$ & no & no & 46,XX,del (15) (q11q13) & 7 & 2,5 & 9,5 \\
\hline 4 & $\mathrm{~F}$ & $1 \mathrm{a} 6 \mathrm{~m}$ & $14 \mathrm{a} 7 \mathrm{~m}$ & no & no & 46,XX,del (15) (q11q13) & 8 & 4,5 & 12,5 \\
\hline 5 & M & $8 m$ & $6 \mathrm{a}$ & no & no & 46,XY,del (15) (q11q13) & 8 & 2 & 10 \\
\hline 6 & M & $1 \mathrm{a}$ & $1 \mathrm{a}$ & no & no & 46,XY,del (15) (q11q13) & 6 & 1,5 & 7,5 \\
\hline 7 & $\mathrm{~F}$ & $8 m$ & $7 \mathrm{a}$ & no & ish del (15)(q11q13)(D15S10-) & 46,XX,del (15) (q11q13) & 8 & 5 & 13 \\
\hline 8 & M & $4 \mathrm{a}$ & $8 \mathrm{a} 9 \mathrm{~m}$ & no & ish del (15)(q11q13)(D15S10-) & 46,XY,del (15) (q11q13) & 8 & 3,5 & 11,5 \\
\hline 9 & M & $7 \mathrm{~m}$ & $7 a$ & A & ish del (15)(q11q13)(D15S10-) & 46,XY,del (15) (q11q13) & 8 & 3,5 & 11,5 \\
\hline 10 & F & $5 \mathrm{~m}$ & $4 \mathrm{a} 6 \mathrm{~m}$ & $\mathrm{~A}$ & ish del (15)(q11q13)(D15S10-) & $46, \mathrm{XX}, \mathrm{t}(13 ; 14)$,del (15) (q11q13) & 3) 8 & 4 & 12 \\
\hline 11 & F & $6 \mathrm{a}$ & 18a & no & ish del (15)(q11q13)(D15S10-) & $46, X X, t(3 ; 15)$ & 8 & 2,5 & 10,5 \\
\hline 12 & $\mathrm{~F}$ & $2 \mathrm{a}$ & $10 \mathrm{a}$ & no & ish del (15)(q11q13)(D15S10-) & $46, \mathrm{XX}$ & 8 & 5 & 13 \\
\hline 13 & F & $4 \mathrm{a}$ & $14 \mathrm{a}$ & no & ish del (15)(q11q13)(D15S10-) & $46, \mathrm{XX}$ & 8 & 3 & 11 \\
\hline 14 & F & $1 \mathrm{~m}$ & $1 \mathrm{a} 11 \mathrm{~m}$ & no & ish del (15)(q11q13)(D15S10-) & $46, X X$ & 6 & 3 & 9 \\
\hline 15 & F & $1 \mathrm{~m}$ & $20 \mathrm{a}$ & $\mathrm{A}$ & ish del (15)(q11q13)(D15S10-) & no & 8 & 4 & 12 \\
\hline 16 & F & $20 \mathrm{a}$ & 23 & A & ish del (15)(q11q13)(D15S10-) & no & 8 & 4,5 & 12,5 \\
\hline 17 & $\mathrm{~F}$ & $18 \mathrm{~m}$ & $18 \mathrm{~m}$ & $\mathrm{~A}$ & ish del (15)(q11q13)(D15S10-) & $46, \mathrm{XX}$ & 6 & 3,5 & 9,5 \\
\hline 18 & M & $2 \mathrm{~m}$ & $2 \mathrm{a} 7 \mathrm{~m}$ & A & ish del (15)(q11q13)(D15S10-) & $46, X Y$ & 6 & 4 & 10 \\
\hline 19 & F & $3 m$ & 3a11m & $\mathrm{A}$ & ish del (15)(q11q13)(D15S10-) & $46, \mathrm{XX}$ & 7 & 4,5 & 11,5 \\
\hline 20 & F & $1 \mathrm{a}$ & $1 \mathrm{a}$ & A & ish del (15)(q11q13)(D15S10-) & $46, X X$ & 7 & 0,5 & 7,5 \\
\hline 21 & M & $6 m$ & $4 \mathrm{a} 11 \mathrm{~m}$ & A & ish del (15)(q11q13)(D15S10-) & $46, X Y$ & 8 & 4 & 12 \\
\hline 22 & M & $1 \mathrm{a} 9 \mathrm{~m}$ & $3 \mathrm{a} 6 \mathrm{~m}$ & $\mathrm{~A}$ & ish del (15)(q11q13)(D15S10-) & $46, X Y$ & 8 & 4,5 & 12,5 \\
\hline 23 & M & $2 a$ & $4 \mathrm{a} 8 \mathrm{~m}$ & A & ish del (15)(q11q13)(D15S10-) & $46, X Y$ & 8 & 2 & 10 \\
\hline 24 & F & $4 a$ & $5 \mathrm{a} 6 \mathrm{~m}$ & A & ish 15q11q13 (D15S10x2) & no & 5 & 2,5 & 7,5 \\
\hline 25 & F & $4 \mathrm{a}$ & $5 \mathrm{a} 6 \mathrm{~m}$ & $\mathrm{~A}$ & ish 15q11q13 (D15S10x2) & no & 5 & 2,5 & 7,5 \\
\hline 26 & F & $3 \mathrm{~m}$ & $3 m$ & A & ish 15q11q13 (D15S10x2) & no & 6 & 1,5 & 7,5 \\
\hline 27 & M & 1a & $17 \mathrm{a}$ & A & ish 15q11q13 (D15S10x2) & no & 8 & 3,5 & 11,5 \\
\hline 28 & M & $7 a$ & $11 \mathrm{a}$ & A & ish 15q11q13 (D15S10x2) & 46,XY/47,XY +mar & 8 & 5 & 13 \\
\hline 29 & M & $10 \mathrm{~d}$ & $2 \mathrm{a} 5 \mathrm{~m}$ & $\mathrm{~A}$ & ish 15q11q13 (D15S10x2) & $46, \mathrm{XY}$ & 6 & 3,5 & 9,5 \\
\hline 30 & $\mathrm{~F}$ & $3 \mathrm{~m}$ & $5 \mathrm{a} 1 \mathrm{~m}$ & A & ish 15q11q13 (D15S10x2) & $46, \mathrm{XX}$ & 7 & 3,5 & 10,5 \\
\hline 31 & F & $3 m$ & $5 \mathrm{a} 1 \mathrm{~m}$ & A & ish 15q11q13 (D15S10x2) & $46, X X$ & 7 & 3,5 & 10,5 \\
\hline 32 & F & $2 \mathrm{a}$ & $6 a$ & A & ish 15q11q13 (D15S10x2) & $46, X X$ & 8 & 4 & 12 \\
\hline 33 & M & $1 \mathrm{~m}$ & $4 \mathrm{~m}$ & A & ish 15q11q13 (D15S10x2) & $46, X Y$ & 6 & 1 & 7 \\
\hline 34 & $\mathrm{~F}$ & $2 \mathrm{~m}$ & $8 \mathrm{a} 3 \mathrm{~m}$ & A & ish 15q11q13 (D15S10x2) & $46, X X$ & 8 & 4,5 & 12,5 \\
\hline 35 & F & $17 \mathrm{a}$ & $29 a$ & $\mathrm{~A}$ & ish 15q11q13 (D15S10x2) & $46, X X$ & 8 & 4 & 12 \\
\hline 36 & F & $1 \mathrm{~m}$ & $1 \mathrm{~m}$ & A & ish 15q11q13 (D15S10x2) & $46, \mathrm{XX}$ & 5 & 1 & 6 \\
\hline 37 & M & $1 \mathrm{~m}$ & $1 \mathrm{~m}$ & A & no & no & 5 & 0,5 & 5,5 \\
\hline 38 & M & $9 a$ & $9 \mathrm{a} 11 \mathrm{~m}$ & A & no & no & 8 & 3,5 & 11,5 \\
\hline 39 & $\mathrm{~F}$ & $1 \mathrm{~m}$ & $5 \mathrm{a} 6 \mathrm{~m}$ & $\mathrm{~A}$ & no & no & 8 & 4 & 12 \\
\hline 40 & F & $7 a$ & $7 a$ & A & no & no & 8 & 4 & 12 \\
\hline 41 & M & $8 \mathrm{~m}$ & $8 \mathrm{~m}$ & A & no & $46, X Y$ & 6 & 1 & 7 \\
\hline 42 & F & $2 \mathrm{a}$ & 21a & $\mathrm{A}$ & no & $46, \mathrm{XX}$ & 8 & 4,5 & 12,5 \\
\hline 43 & M & $5 a$ & $7 \mathrm{a} 10 \mathrm{~m}$ & A & no & $46, \mathrm{XY}$ & 8 & 3,5 & 11,5 \\
\hline 44 & M & $4 \mathrm{a}$ & 8a1m & A & no & $46, X Y$ & 8 & 4,5 & 12,5 \\
\hline 45 & F & $5 a$ & $7 \mathrm{a} 6 \mathrm{~m}$ & $\mathrm{~A}$ & no & $46, \mathrm{XX}$ & 6 & 3 & 9 \\
\hline
\end{tabular}

No: no realizado

A: metilación anormal

ish 15q11q13 (D15S10x2): FISH normal, sin deleción

ish del (15)(q11q13)(D15S10-): FISH alterado, con deleción. 
Tabla 3. Resumen de las características fenotípicas de los 45 pacientes de acuerdo a los criterios de $\mathrm{H}$ olm

\begin{tabular}{|c|c|c|c|c|}
\hline \multirow[t]{2}{*}{ Criterios diagnósticos } & \multicolumn{4}{|c|}{ Edad al momento de aplicar el score } \\
\hline & $\begin{array}{c}<1 \text { año } \\
(\mathrm{N}=7)\end{array}$ & $\begin{array}{c}<3 \text { años } \\
(\mathrm{N}=11)\end{array}$ & $\begin{array}{c}>3 \text { años } \\
(\mathrm{N}=34)\end{array}$ & $\begin{array}{c}\text { Total } \\
(\mathrm{N}=45)\end{array}$ \\
\hline \multicolumn{5}{|l|}{ Criterios mayores } \\
\hline Hipotonía neonatal & $7 / 7$ & $11 / 11$ & $34 / 34$ & $45 / 45$ \\
\hline Problemas de alimentación & $7 / 7$ & $10 / 11$ & $32 / 34$ & $42 / 45$ \\
\hline Ganancia ponderal aumentada & $1 / 7$ & $3 / 11$ & $32 / 34$ & $35 / 45$ \\
\hline Dismorfias faciales & $7 / 7$ & $11 / 11$ & $34 / 34$ & $45 / 45$ \\
\hline Hipogonadismo & $6 / 7$ & $9 / 11$ & $32 / 34$ & $41 / 45$ \\
\hline Retraso del desarrollo & $5 / 7$ & $9 / 11$ & $34 / 34$ & $43 / 45$ \\
\hline Hiperfagia & $1 / 7$ & $1 / 11$ & $30 / 34$ & $31 / 45$ \\
\hline Defecto citogenético o molecular & $7 / 7$ & $11 / 11$ & $34 / 34$ & $45 / 45$ \\
\hline \multicolumn{5}{|l|}{ Criterios menores } \\
\hline Disminución de actividad fetal & $6 / 7$ & $10 / 11$ & $32 / 34$ & $42 / 45$ \\
\hline Problemas conductuales & $0 / 7$ & $2 / 11$ & $24 / 34$ & $26 / 45$ \\
\hline Trastornos del sueño & $0 / 7$ & $1 / 11$ & $11 / 34$ & $12 / 45$ \\
\hline Talla baja & $0 / 7$ & $3 / 11$ & $25 / 34$ & $28 / 45$ \\
\hline Hipopigmentación & $0 / 7$ & $0 / 11$ & $18 / 34$ & $18 / 45$ \\
\hline Manos y pies pequeños & $3 / 7$ & $7 / 11$ & $32 / 34$ & $39 / 45$ \\
\hline Manos angostas & $1 / 7$ & $5 / 11$ & $30 / 34$ & $35 / 45$ \\
\hline Estrabismo/miopía & $2 / 7$ & $4 / 11$ & $9 / 34$ & $13 / 45$ \\
\hline Saliva viscosa & $2 / 7$ & $6 / 11$ & $25 / 34$ & $31 / 45$ \\
\hline Defectos del lenguaje & $0 / 7$ & $2 / 11$ & $27 / 34$ & $29 / 45$ \\
\hline Picking & $0 / 7$ & $2 / 11$ & $26 / 34$ & $28 / 45$ \\
\hline
\end{tabular}

cariograma y prueba de metilación, 6 (13,3\%) FISH y prueba de metilación y 18 (40\%) cariograma, FISH y prueba de metilación.

En 23 pacientes $(51,1 \%)$ se demostró una deleción por citogenética convencional o por FISH, en $13(28,9 \%)$ no existe deleción determinada por FISH y en 9 (20\%) no se ha descartado la existencia de microdeleción pues no se les ha evaluado aún con FISH, los otros 6 pacientes a los que no se les realizó estudio con FISH tienen la deleción detectable en el análisis cromosómico con bandeo de alta resolución.

El puntaje promedio de los individuos SPW en el score para el diagnóstico clínico fue de 8 puntos para los menores de 3 años $(n=11)$ y de 11,5 para los mayores de 3 años $(n=34)$. En los pacientes con edad igual o menor a 12 meses $(n=7)$, el score clínico fue de 7 puntos. En el periodo de recién nacido, todos los pacientes presentaron hipotonía y dificul- tad severa en la alimentación y 90\% hipogonadismo. En los pacientes controles, las características fenotípicas de derivación fueron, en los menores hipotonía y en los mayores obesidad y retraso del desarrollo. El resumen de los puntajes en el score de Holm en estos pacientes se presenta en la Tabla 4. En este grupo, el puntaje promedio del score clínico fue de 4,6 puntos para los menores de 3 años $(n=16), 7,3$ puntos para los mayores de 3 años $(n=7)$ y 4,5 en el grupo de pacientes con edad menor o igual a un año $(n=3)$. Si bien el número de pacientes es pequeño para determinar si hay diferencias estadísticamente significativas, la diferencia entre ambos grupos es evidente.

En el grupo en estudio, al relacionar el puntaje del score clínico con la presencia o no de deleción, el score promedio fue 11 puntos en los individuos con deleción y 10 puntos en los sin deleción. 
Tabla 4. Score de H olm en pacientes con prueba de metilación normal

\begin{tabular}{|c|c|c|c|c|c|c|}
\hline \multirow[t]{2}{*}{ Paciente } & \multirow[t]{2}{*}{ Sexo } & \multirow[t]{2}{*}{ Edad } & \multirow[t]{2}{*}{ Metilación } & \multicolumn{3}{|c|}{ Score de Holm (puntos) } \\
\hline & & & & $\begin{array}{l}\text { Criterios } \\
\text { mayores }\end{array}$ & $\begin{array}{l}\text { Criterios } \\
\text { menores }\end{array}$ & Total \\
\hline 1 & M & $6 a$ & $\mathrm{~N}$ & 4 & 1,5 & 5,5 \\
\hline 2 & M & $15 \mathrm{a}$ & $\mathrm{N}$ & 3 & 1 & 4 \\
\hline 3 & $\mathrm{~F}$ & $6 \mathrm{a}$ & $\mathrm{N}$ & 6 & 3 & 9 \\
\hline 4 & M & 6 a $6 \mathrm{~m}$ & $\mathrm{~N}$ & 4 & 2 & 6 \\
\hline 5 & $\mathrm{M}$ & $5 \mathrm{a}$ & $\mathrm{N}$ & 7 & 4 & 11 \\
\hline 6 & $\mathrm{~F}$ & $9 \mathrm{a}$ & $\mathrm{N}$ & 6 & 4 & 10 \\
\hline 7 & $\mathrm{M}$ & 6 a $6 \mathrm{~m}$ & $\mathrm{~N}$ & 6 & 4 & 10 \\
\hline 8 & $\mathrm{~F}$ & 2 a $4 \mathrm{~m}$ & $\mathrm{~N}$ & 3 & 2,5 & 5,5 \\
\hline 9 & M & 3 a $2 \mathrm{~m}$ & $\mathrm{~N}$ & 4 & 2 & 6 \\
\hline 10 & M & $9 \mathrm{~m}$ & $\mathrm{~N}$ & 4 & 1 & 5 \\
\hline 11 & $\mathrm{~F}$ & $8 \mathrm{a}$ & $\mathrm{N}$ & 4 & 2 & 6 \\
\hline 12 & $\mathrm{~F}$ & $3 \mathrm{a}$ & $\mathrm{N}$ & 4 & 1 & 5 \\
\hline 13 & M & 9 a $9 \mathrm{~m}$ & $\mathrm{~N}$ & 4 & 2,5 & 6,5 \\
\hline 14 & M & $4 \mathrm{a}$ & $\mathrm{N}$ & 4 & 1,5 & 5,5 \\
\hline 15 & $\mathrm{~F}$ & 2 a $9 \mathrm{~m}$ & $\mathrm{~N}$ & 3 & 0,5 & 3,5 \\
\hline 16 & $\mathrm{~F}$ & $12 \mathrm{a}$ & $\mathrm{N}$ & 5 & 5,5 & 10,5 \\
\hline 17 & $\mathrm{~F}$ & $5 \mathrm{a}$ & $\mathrm{N}$ & 4 & 2 & 6 \\
\hline 18 & $\mathrm{~F}$ & $1 \mathrm{a}$ & $\mathrm{N}$ & 4 & 1 & 5 \\
\hline 19 & $\mathrm{~F}$ & $1 \mathrm{a} 1 \mathrm{~m}$ & $\mathrm{~N}$ & 4 & 1 & 5 \\
\hline 20 & M & $11 \mathrm{a}$ & $\mathrm{N}$ & 6 & 3,5 & 9,5 \\
\hline 21 & $\mathrm{~F}$ & $4 \mathrm{~m}$ & $\mathrm{~N}$ & 3 & 0,5 & 3,5 \\
\hline 22 & M & $6 \mathrm{a}$ & $\mathrm{N}$ & 3 & 0 & 3 \\
\hline 23 & $\mathrm{~F}$ & $6 \mathrm{a}$ & $\mathrm{N}$ & 5 & 2,5 & 7,5 \\
\hline
\end{tabular}

Dos pacientes presentaron además otras alteraciones cromosómicas estructurales (46,XX, $\mathrm{t}(13 ; 14)$ y $46, \mathrm{XX}, \mathrm{t}(3 ; 15))$ y uno un marcador cromosómico extra, de origen no precisado. En el total de la muestra se considera un par de gemelas idénticas sin deleción.

\section{DISCUSIÓN}

Todos los pacientes estudiados tienen un fenotipo característico y la alta positividad del score clínico valida su uso en la práctica diaria. El fenotipo es claramente edad dependiente, lo que queda demostrado con la diferencia de score encontrada en los distintos grupos etarios.

Los pacientes con deleción no tienen un score clínico significativamente mayor que los sin dele- ción, por lo que su importancia en la relación genotipo-fenotipo no queda claramente demostrada en este estudio. Es probable que se produzca una mayor diferencia entre los individuos con deleción y con DUP al analizar el fenotipo conductual pues se ha descrito que las características conductuales compulsivas, además de la hiperfagia e incluyendo la psicosis, son más frecuentes en individuos con DUP que en los con deleción ${ }^{13-17}$. Esta condición podría ser evaluada en otros trabajos y escapa a los objetivos de este estudio.

$\mathrm{Si}$ bien la prueba de metilación es suficiente para establecer el diagnóstico, lo ideal es realizar un estudio completo, que incluya siempre cariograma, e idealmente FISH y estudio de microsatélites para el diagnóstico de disomía uniparental con el fin de determinar el origen exacto de la 
afección y entregar un consejo genético certero. Sin embargo, la alta sensibilidad del estudio de metilación hace que se considere el examen de elección para la confirmación diagnóstica de esta afección.

En este estudio, se detectó la presencia de deleción en 51\% del total de individuos evaluados, lo que es un poco menor que $70-75 \%$, descrito por la literatura. Es importante mencionar que si sólo se considera a los pacientes a los que se le realizó FISH $(n=36)$, el porcentaje de casos positivos para la deleción aumenta a $64 \%$. La menor frecuencia con que se encontró la deleción podría corresponder a que falta completar el análisis con FISH en el $9 \%$ de los pacientes estudiados y también a factores propios de la muestra como es el número aún pequeño de sujetos en estudio. La confirmación molecular de DUP a través de estudios de microsatélites $^{18}$ en $29 \%$ de los pacientes en los que se demostró que el SPW no estaba determinado por la deleción característica en 15q11q13, se encuentra actualmente en proceso y será motivo de una próxima publicación.

\section{REFERENCIAS}

1. Buttler MG. Prader-Willi syndrome: Current understanding of cause and diagnosis. Am J Med Genet 1990; 35: 319-32.

2. Differentiated recurrence risk estimations in the Prader-Willi síndrome. Clin Genet 1992; 41: 3038.

3. Buiting K, Saitoh S, Gross S, Dittrich B, Schwartz $\mathrm{S}$, Nichoшs RD ET AL. Inherited microdeletions in the Angelman and Prader-Willi syndromes define an imprinting center on human chromosome 15. Nat Genet 1994; 9: 395-400.

4. GlenN CC, Driscoll DJ, Yang TP, Nichols RD. Genetic imprinting: Potential function and mechanisms revealed by the Prader Willi and Angelman syndromes. Mol Hum Reprod 1997; 3: 321-32.

5. Nichols RD, Knoll JHM, Buttler MG, Karam S, LaLANDE M. Genetic imprinting suggested by maternal heterodisomy in non-deletion Prader-Willi syndrome. Nature 1989; 342: 281-5.

6. Nichoшs RD. Genomic imprinting and uniparental disomy in Angelman and Prader-Willi syndrome:
La clara relación de las características clínicas consideradas en el score de Holm y los puntajes obtenidos a las distintas edades valida su utilización a cualquier edad, incluyendo niños menores de 12 meses.

La asociación de hipotonía e hipogonadismo (hipoplasia de labios menores, clítoris, o ambos, en las niñitas; escroto hipoplásico y criptorquidia en los varones) en el período neonatal, que se observó en alrededor de $90 \%$ de los pacientes estudiados, seńa mandatoria de hacer estudio diagnóstico.

En conclusión, se recomienda que ante la sospecha de SPW, se realice al menos estudio de metilación y cariograma. Con la prueba de metilación se confirma el diagnóstico de SPW y con el estudio cromosómico se pueden descartar otras alteraciones cromosómicas estructurales o numéricas las que cuando están presentes pueden significar un riesgo aumentado de recurrencia de otras alteraciones. Se demuestra la utilidad del score de Holm en la evaluación diagnóstica de pacientes chilenos con SPW por lo que se recomienda su uso en la práctica clínica.

A review. Am J Med Genet 1993; 46: 16-25.

7. CAssidy S. Prader-Willi syndrome. In: Management of Genetic Syndromes. Cassidy S, Allanson J Eds. John Wiley \& sons, Inc., Publication, New York. 2001; 301-22.

8. Ozcelik T, LefF S, Robinson W, Donlon T, Lalande $\mathrm{H}$, SANJINES ET AL. Small nuclear ribonucleoprotein polypeptide $\mathrm{N}$ (SNRPN), an expressed gene in the Prader-Willi syndrome critical region. Nat Genet 1992; 2: 265-9.

9. Holm VA, Cassidy SB, Buttler MG, Hanchett JM, Greenswag LR, Whitman BY et aL. Prader-Willi syndrome: Consensus diagnostic criteria. Pediatrics 1993; 91: 398-402.

10. Gunay-Agun M, Schwartz S, HeEger S, O'Riordan MA, CASSIDY S. The changing purpose of Prader Willi Syndrome clinical diagnostic criteria and proposed revised criteria. Pediatrics 2001; 108 (5):E92.

11. Aumende MA, Curotto B, Santa María L, Aracena M, CoRTÉs F. Prader-Willi syndrome due to 15q11-q13 deletion in a girl with an inherited $(13 ; 14)$ Robertsonian translocation. Am J Med Genet 2002; 113: 307-8. 
12. Santa María L, Curotto B, Contés F, Rojas C, AuEnde MA. Diagnóstico Molecular de los Síndromes de Prader Willi y Angelman: test de metilación, citogenética y FISH. Rev Méd Chile 2001; 129: 367-74.

13. BeARdsmore A, Dorman T, CoOper SA, WebB T. Affective psychosis and Prader-Willi syndrome. J Intellect Disabil Res 1998; 42: 463-71.

14. Prader-Willi syndrome and psychotic symptoms 2. A preliminary study of prevalence using the Psychopathology Assessment Schedule for Adults with Developmental Disability checklist: J Intellect Disabil Res 1998; 42: 451-4.

15. Dykens EM, Leckman JF, CASSidy SB. Maladaptive behavior differences in Prader-Willi syndrome due to paternal deletion versus maternal uniparental disomy. Am J Ment Retard 1999; 104: 67-77.

16. Dykens EM, LeCKMAN JF, CASSIDY SB. Obsessions and compulsions in Prader-Willi syndrome. J Child Psychol Psychiatry 1996; 37: 995-1002.

17. Verhoeven WM, Tuinier S, Curfs LM. Prader-Willi syndrome: the psychopathological phenotype in uniparental disomy. J Med Genet 2003 Oct;40(10):e112.

18. Mutirangura A, Greenberg F, Butler MG, Malcolm S, Nichols RD, Chakravarti A et al. Multiplex PCR of three dinucleotide repeats in the Prader Willi/ Angelman critical region (15q11-q13): molecular diagnosis and mechanism of uniparental disomy. Hum Mol Genet 1993; 2: 143-51. 